\title{
Exotic Heavily Ionizing Particles can be Constrained by the Geological Abundance of Fullerenes
}

\author{
J. I. Collar ${ }^{1,2, *}$ and K. Zioutas ${ }^{3}$ \\ ${ }^{1}$ Groupe de Physique des Solides (UMR CNRS 75-88), Université Paris 7, 2 Pl. Jussieu, Paris 75251, France \\ ${ }^{2}$ EP Division, CERN, CH-1211 Geneve 23, Switzerland \\ ${ }^{3}$ Physics Department, University of Thessaloniki, GR-54006 Thessaloniki, Greece
}

(Received 15 March 1999)

\begin{abstract}
The $\mathrm{C}_{60}$ molecule exhibits a remarkable stability that leads to its survival in ancient carbonaceous rocks initially subjected to the elevated temperature requisite for its formation. Elementary particles having a large electronic stopping power can similarly form $\mathrm{C}_{60}$ and higher fullerenes in their wake. Combined, these two features point at the possibility of using the $\mathrm{C}_{60}$ presence in selected bulk geological samples as a new type of nuclear track detector, with applications in astroparticle physics.

PACS numbers: 95.55.Vj, 61.82.Pv, 78.30.Na, 95.35.+d
\end{abstract}

Sixty carbon atoms, arranged spherically as if on the surface of a soccer ball, form the $\mathrm{C}_{60}$ molecule (Buckminster fullerene). Of all possible formations, this peculiar one has the highest symmetry, lowest energy level, no dangling bonds, and is therefore chemically inert much like a noble gas. This leads to an extraordinary photochemical and thermal stability that fueled the expectations for its abundance in the galaxy [1] soon after its discovery. First searches for $\mathrm{C}_{60}$ in meteorites and the interstellar medium turned up negative [2,3]; the presence of hydrogen as an abundant coreactant in stellar atmospheres was recognized as an inhibiting factor in its production [3]. In spite of this, there is now growing evidence for $\mathrm{C}_{60}$ in interstellar space [4]. On Earth, the confirmation of a natural $\mathrm{C}_{60}$ presence followed a similar chronicle: Only particular geological samples having once withstood the high temperature conditions requisite for $\mathrm{C}_{60}$ formation have so far shown this evidence. This is the case of precambrian shungite [5], the most highly methamorphosed coal known, or fulgurite [6], the glassy product of a lighting strike. Of particular interest is its presence in shock-produced rubble in the 1.85-billion-year-old Sudbury impact crater $[7,8]$ and in samples from CretaceousTertiary $(\mathrm{K} / \mathrm{T})$ boundary clay seams and coals [9]. In the first case, atomic helium encapsulated in the fullerene exhibits an extraterrestrial ${ }^{3} \mathrm{He} /{ }^{4} \mathrm{He}$ ratio, pointing at an origin previous to the impact itself [8]. The high concentration of fullerenes (1-10 ppm) in these samples illustrates the long-term survival of the molecule; the ability to retain extraterrestrial $\mathrm{He}$ exemplifies its endurance of extreme conditions such as those accompanying crater formation. The $\mathrm{C}_{60}$ presence in concentrations typical of soots from common flames in $\mathrm{K} / \mathrm{T}$ samples, but not above or below the boundary, is hypothesized to arise from the raging of postimpact sooting wildfires [9].

In the laboratory, fullerenes have been produced by laser ablation, in carbon arcs, by combustion of benzene and lately by ion beam irradiation $[10,11] . \mathrm{C}_{60}$ and other polycyclic compounds remain in latent tracks of energetic ions in carbonaceous matter, albeit a minimum of a few
$\mathrm{eV} / \AA^{3}$ in transferred electronic energy is needed for this process $[10,12,13]$, so as to permit the completion of chemical formation before the onset of cooling in the particle's hot plasma aftertrack. Given the apparent scarcity of fullerenes in common geological samples, yet its demonstrated ability to survive over long periods, it is natural to wonder if hypothetical highly ionizing and deeply penetrating elementary particles such as magnetic monopoles, nuclearites [14], $Q$ balls [15], etc., should not have left a detectable cumulative signature in ancient carbonaceous rocks. In this Letter, recent models and measurements of $\mathrm{C}_{60}$ production under particle irradiation are employed to estimate its yield from such cosmic exotica, comparing it with that from common terrestrial sources of radiation. This geochemical method is appraised judging from the sensitivity of present-day chromatographic techniques and current efficiency in $\mathrm{C}_{60}$ extraction from bulk minerals.

A condition for the applicability of this technique is that of good fullerene preservation over the age of the rock. There are several precautions to be taken in this respect for sample selection, a situation similar to that of mica searches $[16,17]$ for weakly interacting massive particles (WIMPs) and monopoles, where the thermal history of the mineral must be such that annealing of latent etch tracks is minimized. While $\mathrm{C}_{60}$ integrity under impact and static pressure in the upper layers of the Earth's crust seems assured (it is stable up to 130-170 kbar [18]), there are factors such as exposure to $\mathrm{O}_{3}$ [19] and UV light [20] to be controlled. An environment low in ozone is imperative since the destruction of fullerenes is 5 orders of magnitude faster in the presence of $\mathrm{O}_{3}$ than $\mathrm{O}_{2}$. In fact, the shungite of Ref. [5] is thought to have been protected from $\mathrm{O}_{3}$ oxidation by the coal in which it was contained [19]. The concentration of hydrogen and sulfur in the rock should also be a criterion for selection, the second preventing $\mathrm{C}_{60}$ oxidation when present as sulfide silicates [7]. The thermal decomposition of fullerenes in solid phase, another determining factor, has been measured [21]: The remaining fullerene fraction after a time $t$ at temperature $T$ is 
$\exp [-k(T) t]$, where $k(T)=1.24 \times 10^{9} \exp \left(\frac{-\tau}{T}\right) \mathrm{s}^{-1}$ and $\tau=3.2 \times 10^{4} \mathrm{~K}$, i.e., a $90 \%$ survival probability over $10^{9}$ years even if subjected to a constant $250{ }^{\circ} \mathrm{C}$. Finally, fullerenes exhibit very good stability towards intense irradiation with high-energy electron pulses and gamma rays [22], guaranteeing their resistance to scarce environmental minimum ionizing radiations. In view of these remarkable signs of immunity, the ppm presence (or not) of fullerenes in ancient rocks seems to be less a question of long-term stability and more of sample origin, as experimental findings confirm. If exposure to certain elementary particles over the lifetime of the sample is an abundant source of fullerenes, these should endure to prove the particle's crossing.

It is the ability to efficiently leach fullerenes from bulk mineral and to detect them that ultimately limits this method. In this Letter the state-of-the-art in high-pressure liquid chromatography (HPLC) is conservatively adopted as the detection reference. Similarly, we do not assume a leaching efficiency far beyond what is accomplished nowadays, i.e., that $\mathrm{O}(1) \mathrm{kg}$ samples can be nearly completely demineralized $[9,23]$ and reduced to $\sim 0.1 \mathrm{ml}$ of liquid containing all organic substances of interest. In essence, this procedure consists of pulverization followed by repeated treatment with $\mathrm{HCl} / \mathrm{HF}$ to create a C-rich residue, refluxing of this in a small volume of toluene (a good solvent for aromatic compounds), centrifugation and/or filtration, and concentration of the volume to $0.1-1 \mathrm{ml}$ by evaporation under reduced pressure $[9,23,24]$. The recovery efficiency for thus-treated fullerene-spiked mineral samples has been shown to be $\sim 90 \%$ [23]. At a reasonable signal-to-noise ratio, HPLC is currently able to spot $\sim 10^{-10} \mathrm{~g}$ of fullerene in $0.1 \mathrm{ml}$ toluene aliquots [9,11]: This is the realistic figure adopted below to estimate the present particle detection ability.

The production of fullerenes in the wake of an energetic particle follows a sequence of events [10-13] that begins with the destruction of all molecular bonds in the track core due to the formation of a short-lived zone of highly excited neutral target atoms by the electronic energy transfer from the projectile. This is followed by the nucleation of one-dimensional C-C complexes via random "sticky" collisions of this gas, coalescing into twodimensional pentagonal and hexagonal rings that in turn form closed 3D complexes, and among those fullerenes. The concept of a threshold in electronic stopping power for fullerene formation is interchangeable with that of a minimum track temperature above which condensation is facilitated [13]. The dependence of the track temperature $T(r, t)$ on the axial distance $r$ from the particle path and time $t$ after passing is [25]

$$
T(r, t)=\frac{T_{0}}{1+\left(4 D t / r_{0}^{2}\right)} \exp \left\{-\frac{\left(r / r_{0}\right)^{2}}{1+\left(4 D t / r_{0}^{2}\right)}\right\},
$$

where $D$ is the thermal diffusivity of the medium and $r_{0} \sim 15 \AA$ is the core radius, which delimits a cylindrical region of plasmalike conditions and highest energy density [25]. The initial track temperature is given by $T_{0}=\alpha S_{e} /\left(\pi \rho C_{V} r_{0}^{2}\right)$, with $\rho$ as the density, $C_{V}$ the specific heat, $S_{e}$ the electronic stopping power, and $\alpha \sim 0.1$ as the fraction of energy going into thermal excitation of the Maxwellian gas of carbon atoms. Leaving aside a dependence on the probability of sticky C-C collisions and on the average density of the gas (both temperature independent to first approximation $[13,26])$, the $\mathrm{C}_{60}$ yield is proportional to

$$
Y_{60} \propto \iiint \Omega(r, T) 2 \pi r d r d z d t
$$

( $z$ denotes the direction along the trajectory). This expression gives the time-integrated volume of material available for fullerene condensation under the favorable conditions described by $\Omega(r, T)$, namely,

$\Omega(r, T)=\left\{\begin{array}{cc}1 \text { for } T_{\min }<T(r, t)<T_{\max }, & 0 \text { elsewhere, } \\ 0 \text { for } r<r_{60}, & 1 \text { elsewhere, }\end{array}\right.$

where the minimum temperature for fullerene formation is estimated at $T_{\min } \sim 750-800 \mathrm{~K}$ in studies of laser ablation of polymers [12,26], and unconfined $\mathrm{C}_{60}$ is seen to readily disintegrate at $T_{\max } \sim 6000 \mathrm{~K}$. The second condition in $\Omega(r, T)$ imposes that the heated volume must be able to physically accommodate the $\mathrm{C}_{60}$ cage within; $r_{60}$ should then naively correspond to the molecule's radius, $3.6 \AA$. A more adequate phenomenological value $r_{60} \sim 9 \AA$ [13] is adopted here.

In the present analysis, $T(r, t)$ must be evaluated numerically to compute $Y_{60}$ for different types of radiation. These yields are in arbitrary units until a normalization point is applied; of the handful of recent irradiations of carbonaceous materials with heavy ions, the results of Fink et al. [11] using $3 \mathrm{GeV} \mathrm{U}^{20+}$ on thin plates of sucrose $\left(S_{e} \sim 2.0 \times 10^{4} \mathrm{keV} / \mu \mathrm{m}\right.$, ion range $\left.\sim 160 \mu \mathrm{m}\right)$ are particularly helpful for this purpose, since the total dissolution of sugar in HPLC reagents guarantees an excellent $\mathrm{C}_{60}$ recovery. The found value of $84 \pm 31$ $\mathrm{C}_{60}$ molecules per incident ion should then reflect the actual $Y_{60}$ closely. Equation (2) can be used to assess $Y_{60}$ if the target is instead a carbonaceous rock: The different stopping powers [27] and parameter values for sucrose $\left[C_{V} \sim 1.2 \times 10^{3} \mathrm{~J} /(\mathrm{kg} \mathrm{K}), D \sim 2.9 \times\right.$ $\left.10^{-7} \mathrm{~m}^{2} / \mathrm{s}, \rho \sim 1.6 \times 10^{3} \mathrm{~kg} / \mathrm{m}^{3}\right]$ and coal-like samples $\left[C_{V} \sim 700 \mathrm{~J} /(\mathrm{kg} \mathrm{K}), D \sim 1.8 \times 10^{-7} \mathrm{~m}^{2} / \mathrm{s}, \rho \sim 2 \times\right.$ $10^{3} \mathrm{~kg} / \mathrm{m}^{3}$ ] translate into an enhancement in $Y_{60}$ by a factor 3.8. The nearly constant $S_{e}$ over the initial $90 \%$ of the ion range in both situations allows one to conclude that, to a good approximation, $\frac{\partial Y_{60}}{\partial z} \simeq 2.5 \times 10^{-4} \mathrm{C}_{60} / \AA$ at $S_{e} \simeq 2.4 \times 10^{4} \mathrm{keV} / \mu \mathrm{m}$ for C-rich rocks (the units of $S_{e}$ and value of $\rho$ are maintained tacitly hereafter). This normalization point anchors our calculation of $\frac{\partial Y_{60}}{\partial z}$ vs $S_{e}$ (shown in Fig. 1). Fink et al. observed that $\mathrm{C}_{60}$ production was a highly inefficient process ( $36 \mathrm{MeV}$ invested per $\mathrm{C}_{60}$ when the molecule's formation energy is $444 \mathrm{eV}$ ). An interpretation $[11,28]$ is that the carbon atoms are highly 


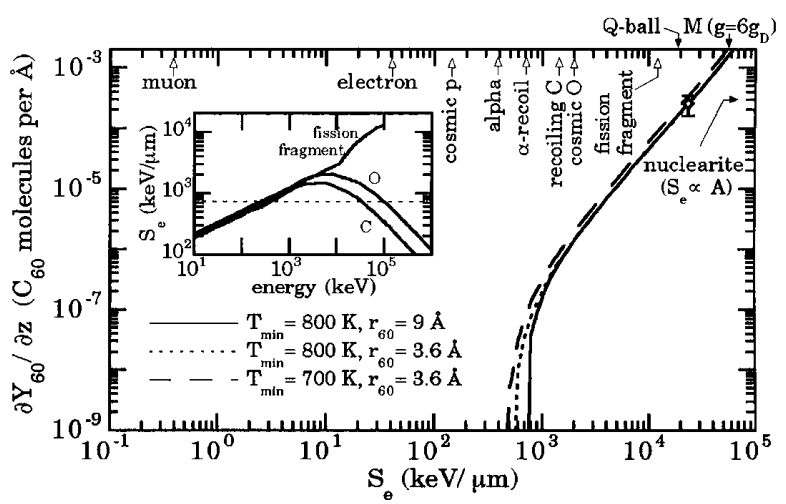

FIG. 1. Estimated $\mathrm{C}_{60}$ yield per unit path length vs particle electronic stopping power, in coal-like rocks. The single point corresponds to the overall normalization based on [11]. The dependence on $T_{\min }$ and $r_{60}$ is shown; similar variations of $r_{0}$ and $T_{\max }$ have a comparatively negligible effect. The slope at high $S_{e}$ is in good agreement with [13]. The maximum $S_{e}$ for common natural sources of radiation is indicated (white arrows) and illustrated in the inset for those above the fullerene production threshold $S_{e} \sim 750 \mathrm{keV} / \mu \mathrm{m}$.

volumetrically diluted in sucrose (one in four), reducing the probability of C-C collisions; this is aggravated by the presence of hydrogen, which can lead to aromatic hydrocarbons rather than fullerenes as stable end products [3]. There should then be an additional enhancement in $Y_{60}$ for rocks primarily composed of $\mathrm{C}$. For these the overall normalization of Fig. 1 is probably largely conservative and not a substitute for calibrations using ions of stopping power similar to that of the particle of interest (calibrations that should also determine the leaching efficiency for a specific rock type).

Do natural backgrounds give rise to a significant fullerene presence in shallow-mined carbonaceous rocks? Isolated minimum ionizing radiations cannot play a role (Fig. 1). However, recoiling carbon nuclei with energies $\geq 30 \mathrm{MeV}\left(Y_{60} \simeq 0.1\right)$ can be induced by underground muons, albeit the cross section for this process [29] is small at $<10^{-3} \mathrm{mb}$. With a muon flux $10^{-3} \mathrm{~cm}^{-2} \mathrm{~s}^{-1}$ at a modest $150 \mathrm{~m}$ depth, these recoils should produce a meager $<10^{-21}\left(\mathrm{~g} \mathrm{C}_{60} / \mathrm{kg}\right.$ rock $) / \mathrm{yr}$. A comparable contribution, if any, is expected from $\mathrm{O}(1) \mathrm{MeV}$ recoils $\left(Y_{60} \simeq 10^{-3}\right)$ originating in elastic scattering of the $2 \times 10^{-7} \mathrm{~cm}^{-2} \mathrm{~s}^{-1}$ fast neutron flux typical below this depth. Similarly, recoiling daughters from $(n, p),(n, \alpha),(\alpha, p)$, and $(\alpha, n)$ reactions in $\mathrm{C}$ can be shown to contribute negligibly, and the same applies to the scarce penetrating medium- and large-mass cosmic ray nuclei. The biggest offenders are, by far, spontaneous fission fragments $\left(Y_{60} \simeq 5\right)$ which result in $2.8 \times 10^{-18}\left(\mathrm{~g} \mathrm{C}_{60} / \mathrm{kg}\right.$ rock $) / \mathrm{yr}$ per ppm ${ }^{238} \mathrm{U}$, making a low $\mathrm{U}$ concentration necessary if ancient samples of age $\sim 1 \mathrm{Gyr}$ are to be utilized (US coals exist with $<0.02 \mathrm{ppm} \mathrm{U}$ ). This is again reminiscent of WIMP mica searches, where $\leq 10^{-4} \mathrm{ppm} \mathrm{U}$ are desirable.

The central result of our analysis is that heavily ionizing particles energetic enough to maintain a constant $S_{e}>$
$10^{3}$ through the Earth's upper crust should produce at least $\sim 2.05 \times 10^{-26} S_{e}^{2.1} \mathrm{~g} \mathrm{C}_{60}$ per cm traversed in C-rich rock. Assuming a spherical rock geometry, with average trajectory length equal to its radius, the limit on their flux attainable by processing a rock mass $M(k g)$ of age $t(\mathrm{yr})$ is then

$$
\begin{aligned}
\Phi\left(\mathrm{cm}^{-2} \mathrm{~s}^{-1} \mathrm{sr}^{-1}\right)<\max [ & 8.2 \times 10^{3} \varepsilon /\left(M t S_{e}^{2.1}\right), \\
8.3 & \left.\times 10^{-12} M^{-2 / 3} t^{-1}\right],
\end{aligned}
$$

where $10^{-10} \varepsilon \mathrm{g}$ is the minimum chromatographically detectable $\mathrm{C}_{60}$ mass $\left(\varepsilon_{\mathrm{HPLC}}=1\right)$. The first term judges the ability of the particles to produce enough fullerenes during the lifetime of the sample, whereas the second imposes that at least one such particle must have crossed it (i.e., dictates the minimum exposure $M t$ necessary for the first term to apply). For magnetic monopoles, this method is essentially applicable only to those with $\beta>10^{-1}$, for which the main mode of energy loss is via ionization. In this sense, this proposal is complementary to mica searches [17], where the sensitivity decreases rapidly above $\beta \sim 10^{-3}$. For $\beta=1$, monopole energy losses in C-rich rock depend on their magnetic charge $g=n g_{D}$ via $S_{e} \simeq 1.6 \times 10^{3} n^{2}[30]\left(g_{D}=\right.$ Dirac charge $)$. Inspection of Eq. (4) shows that, for $n>3, t \sim 10^{9}$, and $M<50$, an improvement of the best current limits [31] in this velocity range is already within reach. For $n=1,2$, a decrease in $\varepsilon$ must be awaited: Using a reasonable $M<100$, future or alternative $\mathrm{C}_{60}$ detection techniques can ameliorate the present experimental limits for grand unified theory fast monopoles $\left(\Phi<3 \times 10^{-16}\right)$ by several orders of magnitude before running into the limitations expressed by the second term in Eq. (4), with the attainable radiopurity of the rock as the only constraint.

Coherent states of squarks and sleptons, predicted by supersymmetric generalizations of the standard model and dubbed $Q$ balls [15], are also expected to be highly penetrating and to have a flux of $\sim 1.25 \times 10^{3} Q_{B}^{-3 / 4}(1 \mathrm{TeV} / \mathrm{m}) \mathrm{cm}^{-2} \mathrm{~s}^{-1}$ if they play a significant role as galactic dark matter [32]. Their mass, $m$, is assumed to be in the $0.1-100 \mathrm{TeV}$ range, and their baryon number must respect $Q_{B}>10^{15}(\mathrm{~m} / 1 \mathrm{TeV})^{4}$. Their stopping power in matter of density $\rho$ should be $\sim 10^{4} \frac{\rho}{1 \mathrm{~g} / \mathrm{cm}^{3}} \mathrm{keV} / \mu \mathrm{m}$, but the energy loss mechanism depends on their being charged or neutral [32]. Here it is conservatively assumed that only charged $Q$ balls are able to provide a dense enough energy transfer to form fullerenes. In that case, a modest reduction in $\varepsilon$ would bring about a challenge to the $Q$ ball sensitivity of large underground detectors such as super-Kamiokande $\left(\right.$ surface area $\left.=7.5 \times 10^{7} \mathrm{~cm}^{2}\right)$.

Stable or metastable condensates of up, down, and strange quarks (a.k.a. strange quark matter or "nuclearites"), with mass numbers ranging from small nuclei to neutron stars $\left(A \sim 10^{56}\right)$, may constitute the ground state of hadronic matter [14,33]. If they make up an important fraction of the galactic dark matter halo, their local 
flux should be $\Phi_{\mathrm{SDM}}=6 \times 10^{5} A^{-1}$ [14]. Their stopping power in rock $\left[4 \times 10^{-6} A^{2 / 3}\left(\frac{v}{300 \mathrm{~km} / \mathrm{s}}\right)^{2} \mathrm{keV} / \mu \mathrm{m}\right.$ for $A>10^{15}$ ] is so enormous that the associated shock wave could in some cases create a visible scar of melted material, an "astroblem" [14] (for large $A$ the electronic and nuclear temperatures in the shock wave are assumed to balance out, making $S_{e}$ a sizable fraction of the total stopping power). An interesting peculiarity of the method proposed here becomes evident in searches for nuclearites: Other techniques (mica, plastic track detectors, scintillators) rely on the principle "one particle, one event," and therefore their flux limits are blind to the value of $A$ (i.e., of $S_{e}$ ). In contrast to this, Eq. (4) improves as $A^{-2.1 \times(2 / 3)}$ for nuclearites. Keeping in mind that $\Phi_{\mathrm{SDM}} \propto A^{-1}$, this means that a negative $\mathrm{C}_{60}$ search could, in principle, exclude nuclearites as the galactic dark matter for all values $A>10^{13}\left(A>10^{25}\right.$ being not yet ruled out). Needless to say, this ability is maimed by the second term in Eq. (4). All the same, such a search can immediately improve the existing sensitivity to these particles (a limit of $\Phi<10^{-20}$ for $A>10^{14}$ ) by 2 orders of magnitude after processing a realistic $M \sim 100$. The possibility that lighter aggregates of strange matter ("strangelets") may reach deeply into the atmosphere [34], a hypothesis put forward to explain several anomalous ("Centauro") events in detectors, can also be tested in this way (strangelet stopping power in rock is expected to weigh in at $\sim 3.5 \times 10^{4} \mathrm{keV} / \mu \mathrm{m}$ [14]).

In conclusion, an ubiquitous abundance of $\mathrm{C}_{60}$ in rocks could be the signature of any new family of energetic highly ionizing elementary particles. Its absence would allow in most cases to further push existing experimental limits, no small feat given their severity. In view of the incipience of studies of fullerene production by ion beams, our conservative estimates should be taken at face value [e.g., temperature- and material-dependent effects $[13,35]$ can increase the exponent of $S_{e}$ in Eq. (4), resulting in an enhanced signal-to-(fission)noise ratio for this search]. Irradiations of $\mathrm{C}$-rich rocks to measure the production/recovery of fullerenes are then in order, together with searches for a natural fullerene presence correlated to spontaneous fission of ${ }^{238} \mathrm{U}$ (possibly a new tool in geological dating).

We are indebted to P. B. Price for a critical reading of the manuscript.

*Corresponding author.

Email address: Juan.Collar@cern.ch

[1] H. W. Kroto and M. Jura, Astron. Astrophys. 263, 275 (1992).

[2] I. Gilmour et al., Lunar Planet. Sci. XXII, 445 (1991).

[3] M.S. de Vries et al., Geochim. Cosmochim. Acta 57, 933 (1993).
[4] B. H. Foing and P. Ehrenfreund, Astron. Astrophys. 317, L59 (1997); F. R. diBrozzolo et al., Nature (London) 369, 37 (1994).

[5] P.R. Buseck et al., Science 257, 215 (1992); O. I. Kon'kov et al., Phys. Solid State 36, 1685 (1994).

[6] T. K. Daly et al., Science 259, 1599 (1993).

[7] L. Becker et al., Science 265, 642 (1994).

[8] L. Becker et al., Science 272, 249 (1996).

[9] D. Heymann et al., Geochim. Cosmochim. Acta 58, 3531 (1994); D. Heymann et al., Science 265, 645 (1994); P. H. Fang and R. Wong, Mater. Res. Innovat. 1, 130 (1997).

[10] L. T. Chadderton et al., Nucl. Instrum. Methods Phys. Res., Sect. B 91, 71 (1994); L. T. Chadderton et al., Radiat. Eff. Defects Solids 127, 163 (1993).

[11] D. Fink et al., Nucl. Instrum. Methods Phys. Res., Sect. B 117, 134 (1996).

[12] E. G. Gamaly and L. T. Chadderton, Proc. R. Soc. London A 449, 381 (1995).

[13] I. S. Bitenski et al., Nucl. Instrum. Methods Phys. Res., Sect. B 82, 356 (1993).

[14] A. de Rújula and S.L. Glashow, Nature (London) 312, 734 (1984); A. de Rújula, Nucl. Phys. A434, 605c (1985).

[15] S. Coleman, Nucl. Phys. B262, 263 (1985).

[16] D. Snowden-Ifft et al., Phys. Rev. Lett. 74, 4133 (1995); J. I. Collar and F. T. Avignone III, Nucl. Instrum. Methods Phys. Res., Sect. B 95, 349 (1995).

[17] P. B. Price and M. H. Salamon, Phys. Rev. Lett. 56, 1226 (1986); R. L. Fleischer et al., Phys. Rev. 184, 1398 (1965).

[18] C.S. Yoo and W. J. Nellis, Science 254, 1489 (1991); J. E. Fisher et al., Science 252, 1288 (1991).

[19] L.P. F. Chibante et al., Carbon 31, 185 (1993); L.P.F. Chibante and D. Heymann, Geochim. Cosmochim. Acta 57, 1879 (1993).

[20] R. Taylor et al., Nature (London) 351, 277 (1991).

[21] S. D. Leifer et al., Phys. Rev. B 51, 9973 (1995) [note the missing minus sign in the exponential of Eq. (2)].

[22] D. K. Maity et al., J. Chem. Soc. Faraday Trans. 89, 95 (1993).

[23] D. Heymann et al., J. Chromatogr. A 689, 157 (1995).

[24] W.S. Wolbach and E. Anders, Geochim. Cosmochim. Acta 53, 1637 (1989).

[25] A. Mozumder, Adv. Radiat. Chem. 1, 1 (1969); R. Macfarlane and D. Torgerson, Phys. Rev. Lett. 36, 486 (1976).

[26] W. R. Creasy and J. T. Brenna, J. Chem. Phys. 92, 2269 (1990); W. R. Creasy, J. Chem. Phys. 92, 7223 (1990).

[27] J.F. Ziegler et al., The Stopping and Range of Ions in Solids (Pergamon Press, Oxford, 1985).

[28] D. Fink et al., Appl. Phys. A 64, 61 (1997).

[29] J.D. Jackson, Classical Electrodynamics (Wiley, New York, 1975), p. 619.

[30] J. Derkaoui et al., Astropart. Phys. 9, 173 (1998).

[31] S. Orito et al., Phys. Rev. Lett. 66, 1951 (1991).

[32] A. Kusenko et al., Phys. Rev. Lett. 80, 3185 (1998).

[33] J. Madsen, in Hadrons in Dense Matter and Hadrosynthesis, edited by J. Cleymans (Springer-Verlag, Berlin, 1999).

[34] G. Wilk and Z. Wlodarczyk, hep-ph/9606401; Nucl. Phys. Proc. Suppl. B52, 215 (1997).

[35] G. Brinkmalm et al., Phys. Rev. B 47, 7560 (1993). 